\title{
Mostruosità e desiderio metafisico: il tema del ragno nell'opera di Victor Hugo
}

\section{Federica Casini}

\section{Q OpenEdition \\ 1 Journals}

\section{Edizione digitale}

URL: http://journals.openedition.org/studifrancesi/29833

DOI: 10.4000/studifrancesi.29833

ISSN: 2421-5856

\section{Editore}

Rosenberg \& Sellier

\section{Edizione cartacea}

Data di pubblicazione: 1 avril 2006

Paginazione: 35-42

ISSN: 0039-2944

\section{Notizia bibliografica digitale}

Federica Casini, «Mostruosità e desiderio metafisico: il tema del ragno nell'opera di Victor Hugo», Studi Francesi [Online], 148 (XLX | I) | 2006, online dal 30 novembre 2015, consultato il 19 avril 2021. URL: http://journals.openedition.org/studifrancesi/29833; DOI: https://doi.org/10.4000/studifrancesi. 29833

\section{(c) (i) $\odot$}

Studi Francesi è distribuita con Licenza Creative Commons Attribuzione - Non commerciale - Non opere derivate 4.0 Internazionale. 


\section{Mostruosità e desiderio metafisico: il tema del ragno nell'opera di Victor Hugo}

Il ragno è l'insetto simbolo della maledizione che per Victor Hugo grava non solo sugli uomini ma anche sugli altri esseri (animati e apparentemente inanimati) ${ }^{1}$ che popolano il cosmo, come animali e piante. Il ragno è il capro espiatorio reietto e aborrito dalla comunità universale. Ritenerlo una vittima animale qualsiasi, tra gli innumerevoli "misérables" (civetta ${ }^{2}$, rospo, maiale, asino etc) che affollano l'opera di Hugo, significherebbe sminuire la centralità di un personaggio che costituisce un vero e proprio tema all'interno della creazione artistica (letteraria e grafica ${ }^{3}$ ) dell'autore.

La figura del ragno compare ben presto nella produzione hugoliana. Una delle prime apparizioni dell'insetto si registra infatti nel romanzo giovanile Bug-Jargal (la cui stesura primitiva risale al 1818) dove il ragno è associato al male, nella fattispecie al perfido nano Habibrah, le cui gambe «se repliaient sous lui comme les bras d'une araignée» ${ }^{4}$. Qualche anno più tardi è l'essere orrendo che tesse la sua tela nella cella dei condannati alla pena capitale in Le dernier jour d'un condamné. Il detenuto rimuove l'«énorme toile d'araignée»" tesa all'angolo del muro, dove sono riportati i nomi di celebri criminali morti sul patibolo ed è preda di una terrificante allucinazione. La brusca impressione che il passaggio del ragno provoca sul suo corpo strappa il detenuto all'incubo e lo riporta alla realtà ${ }^{6}$. L'insetto è accostato in quest'occasione al carcere e alla morte e, sebbene legato a immagini di terrore, ha il merito di esercitare una funzione positiva sul «Condamné».

Alle soglie degli anni Trenta il ragno non ha ancora assunto una compiuta fisionomia vittimaria. Più che in veste di personaggio, di caper emissarius, esso ricorre come presenza simbolica negativa che troverà il suo più compiuto sviluppo in Notre-Dame de Paris (la cui prima edizione è del 1831). Qui «l'araignée hideuse» ${ }^{7}$ è l'emblema della fatalità, dell'ananké; è l'oggetto della contemplazione di Frollo che osserva ammaliato il dramma della mosca impigliata nella tela, metafora esistenziale della vicenda della danzatrice Esmeralda e dello stesso arcidiacono:

Elle vole, elle est joyeuse, elle vient de naittre; elle cherche le printemps, le grand air, la liberté; oh! oui, mais elle se heurte à la rosace fatale, l'araignée en sort, l'araignée hideuse! Pauvre danseuse! pauvre mouche prédestinée! Maître Jacques, laissez faire! c'est la fatalité!

(1) Secondo la teosofia hugoliana (che mescola animismo e reincarnazione punitiva) «tout, bête, arbre et roche» è «vivant sur terre» (Ce que dit la Bouche d'Ombre, v 291, in V. Hugo, Les Contemplations, Paris, Livre de Poche, 1985, p. 494).

(2) In difesa di questi animali, banditi e perseguitati dagli uomini, Hugo compose appassionati versi. Alla civetta, inchiodata alla porta di una casa come Cristo sulla croce, è consacrata la poesia delle Contemplations intitolata La chouette. Il rospo «chassieux» ma indifeso, torturato da un gruppo di monelli, è il protagonista della celebre poesia della Légende des Siècles dal titolo Le Crapaud, in cui è esaltata in termini eroici la figura dell'asino, il «forçat» che, stremato dalla fatica e dalle frustate dell'asinaio, evita di schiacciare, col peso del carro che spinge, il povero rospo agonizzante sulla carreggiata. Asino che diverrà il soggetto di un intero poema: l'Ane. Un «porc fétide», sgozzato vivo da un macellaio e fuggito con orrore dalla comunità di Bagdad compare infine in Sultan Mourad, altra poesia della Légende des Siècles dedicata a una «bête impure».

(3) Celebre Vianden à travers une toile d'araignée, immagine di una ragnatela disegnata da Hugo su un album durante il soggiorno in Lussemburgo del 1871 («J'ai dessiné sur mon livre de voyage la grande toile d'araignée à travers laquelle on aperçoit la ruine de Vianden comme un spectre». Vedi: J.-É. MulleR, Victor Hugo au Luxembourg: vues et visions, Luxembourg, Institut Grand-Ducal, section arts et lettres, 1982).

(4) V. Hugo, Bug Jargal, in Le Dernier Jour d'un Condamné précédé de Bug-Jargal, Paris, Gallimard, 1970 , p. 39.

(5) V. Hugo, Le Dernier Jour d'un Condamné, Paris, Gallimard, 1970, p. 293.

(6) Ivi, pp. 293-295. 
- Hélas! Claude, tu es l'araignée. Claude, tu es la mouche aussi! - Tu volais à la science, à la lumière, au soleil, tu n'avais souci que d'arriver au grand air, au grand jour de la vérité éternelle; mais, en te précipitant vers la lucarne éblouissante qui donne sur l'autre monde, sur le monde de la clarté, de l'intelligence et de la science, mouche aveugle, docteur insensé, tu n'as pas vu cette subtile toile d'araignée tendue par le destin entre la lumière et toi, tu t'y es jeté à corps perdu, misérable fou, et maintenant tu te débats, la tête brisée et les ailes arrachées, entre les antennes de fer de la fatalité! -Maitre Jacques! maître Jacques! laissez faire l'araignée ${ }^{8}$.

L'immagine del combattimento tra il ragno e la mosca sarà utilizzata ancora in Les Travailleurs de la Mer (1866) per raffigurare la lotta tra Gilliatt e la piovra, mostro marino definito «arachnide par la forme»" «Gilliatt avait enfoncé son bras dans le trou; la pieuvre l'avait happé. Elle le tenait. Il était la mouche de cette araignée» ${ }^{10}$.

La figura del ragno in Notre-Dame de Paris assume ugualmente forti connotazioni negative durante l'interrogatorio di Esmeralda in camera di tortura. Nella visione che assale la povera gitana l'insetto è paragonato, insieme ai pipistrelli e ai millepiedi, agli spaventosi arnesi presenti nella «chambre de la question»:

Il lui sembla voir se mouvoir et marcher de toutes parts vers elle, pour lui grimper le long du corps et la mordre et la pincer, tous ces difformes outils de la torture qui étaient, parmi les instruments de tout genre qu'elle avait vus jusqu'alors, ce que sont les chauves-souris, les millepieds et les araignées parmi les insectes et les oiseaux ${ }^{11}$.

L'assimilazione definitiva del ragno a simbolo del supplizio, del male e della morte si registra nelle scene finali del romanzo, quando Frollo osserva il «groupe épouvantable de l'homme et de la jeune fille, de l'araignée et de la mouche» ${ }^{12}$. Il ragno è il boia che decreta sul patibolo la fine della mosca Esmeralda.

In La Trompette du Jugement (poesia inclusa nella prima serie della Légende des Siècles, pubblicata nel 1859) Hugo aveva posto in rapporto diretto il ragno con il diavolo: «Et l'on voyait, au fond de la rondeur obscure / La toile d'araignée horrible de Satan» ${ }^{13}$.

Un accostamento analogo si ritrova in Les Travailleurs de la Mer, dove le «maisons visionnées» delle isole della Manica, barricate e abbandonate dagli uomini a causa delle visite notturne del demonio, pullulano di ragni: «L'épaississement des toiles pleines de mouches indique la paix profonde des araignées. Quelquefois on aperçoit un pot cassé sur une planche. C'est là une maison "visionnée”. Le diable y vient la nuit» ${ }^{14}$.

(7) V. Hugo, Notre-Dame de Paris, Paris, Garnier-Flammarion, 1967, p. 298.

(8) Ivi, pp. 298-299.

(9) V. Hugo, Les Travailleurs de la Mer, Paris, Garnier-Flammarion, 1980, p. 495.

(10) Ivi, p. 502. Il ragno e la piovra costituiscono per Charles Baudouin due simboli della «Mère terrible» e della «Fatalité» (C. BAudouin, Psychanalyse de Victor Hugo, Genève, Éditions du Mont-Blanc, 1943. Nuova edizione: Paris, Armand Colin, 1972, pp. 157-181). Non a caso Hugo definisce Notre-Dame de Paris il romanzo dell'«ananké des dogmes», della fatalità della religione e Les Travailleurs de la Mer il romanzo dell'«ananké des choses», della fatalità della natura (Les Travailleurs de la Mer, $\mathrm{p}$ 107). Le analogie tra la piovra e il ragno, esaminate da una diversa prospettiva, sono state oggetto dell'analisi di Roger Caillois (R. CaIllois, La Pieuvre, essai sur la logique de l'imaginaire, Paris, La Table Ronde, 1973, pp. 219-223).

(11) V. Hugo, Notre-Dame de Paris, p. 331.

(12) Ivi, p. 504

(13) V. Hugo, La Trompette du Jugement, vv. 164-165, in La Légende des Siècles, Paris, GarnierFlammarion, 1967, II, p. 407.

(14) V. Hugo, Les Travailleurs de la Mer, p. 115. 
Ne L'Homme qui rit (1869) il ragno è avvicinato alla sensualissima ed «infernale Josiane»: «Au centre de la toile, à l'endroit où est d'ordinaire l'araignée, Gwynplaine aperçut une chose formidable, une femme nue» ${ }^{15}$.

Nei Misérables (1862) l'aracnide assume connotazioni ambivalenti: da una parte simboleggia i perfidi aguzzini di Cosette, «mouche servant des araignées» presa in trappola nella tela della «gargote Thénardier» ${ }^{16}$; dall'altra appare immagine di salvezza nel momento in cui il forzato Jean Valjean salva un marinaio che aveva perso l'equilibrio rischiando di cadere in acqua: «on eut dit une araignée venant saisir une mouche; seulement ici l'araignée apportait la vie et non la mort $\gg^{17}$.

Esiste poi una serie di poemi degli anni Cinquanta e Sessanta in cui si stabilisce, fa notare P. Albouy ${ }^{18}$, un legame in termini di corrispondenza-opposizione tra il ragno e la stella. Il ragno è un nucleo che emana influssi negativi in modo simile alla stella, centro, al contrario, di irradiazioni benefiche. In un passo della Fin de Satan del 1854 Hugo parla dei misfatti dell'umanità antidiluviana, che hanno liberato un vapore nero ${ }^{19}$. Questa nebbia ha oscurato l'azzurro del cielo che «perdait ses purs rayons, Et par instants semblait plein de hideuses toiles / Où l'araignée humaine avait pris les étoiles» ${ }^{20}$.

Già nel poema Saturne del 1839 Hugo aveva avvicinato «cette planète maléfique ${ }^{21}$ a un ragno al centro della tela: «Ainsi qu'une araignée au centre de sa toile, / Il tient sept lunes d'or qu'il lie à sept essieux ${ }^{22}$. Nella poesia Magnitudo Parvi, risalente agli inizi del $1855^{23}$, il ragno è definito «hydre étoilée, / Au centre du mal se tenant $\gg^{24}$.

Parallelamente i rapporti di ostilità tra il ragno e la stella sembrano attenuarsi in altre poesie, dove a risaltare sono soprattutto le somiglianze tra l'astro e l'aracnide.

Le analogie esistenti tra l'insetto «soleil noir du mal» ${ }^{25}$ e la stella si fanno strette nel poema VIII del libro III delle Contemplations, dove Hugo afferma che «Tout est plein de jour, même la nuit; / Et tout ce qui travaille, éclaire, aime ou détruit, / A des rayons: la roue au dur moyen, l'étoile, / La fleur, et l'araignée au centre de sa toile» ${ }^{26}$.

Il ragno, osserva Albouy, è un essere del male ma possiede i raggi, un tipico attributo del bene che lo apparenta alla stella e ne rende possibile una futura riabilitazione: «De fait, la rédemption se prépare ${ }^{27}$.

Nel 1854 lo spettro di Ce que dit la Bouche d'Ombre invita a piangere su «l'araignée immonde» ${ }^{28}$. L'atteggiamento del poeta nei confronti del tenebroso ragno pare significativamente mutato rispetto agli anni Trenta. Il ripugnante insetto, simbolo della fatalità, è divenuto addirittura un essere da compiangere. Il ragno infatti non è condannato per l'eternità. Esso, come tutti i mostri e le creature malvage, avrà la possibilità di redimersi poiché nella complessa teosofia hugoliana anche il male un giorno si ricongiungerà al bene. Così «On verra $[. .$.$] / L'araignée éclatante$ au seuil des bleus pilastres, / Luire, et se redresser, portant des épis d'astres, / La

(15) V. Hugo, L'Homme qui rit, Paris, GarnierFlammarion, 1982, II, p. 206.

(16) V. Hugo, Les Misérables, Paris, Garnier-

Flammarion, 1967, I, p. 415.

(17) Ivi, I, p. 405.

(18) P. Albouy, La création mythologique chez Victor Hugo, (deuxième édition) Paris, José Corti, 1985, p. 169.

(19) Ibidem.

(20) V. Hugo, La Fin de Satan, Paris, Gallimard, 1984 , p. 48.

(21) P. Albouy, La création mythologique chez Victor Hugo, p. 169.

(22) V. Hugo, Saturne, vv 69-70, in Les Contem- plations, Paris, Livre de Poche, 1985, p. 164.

(23) P. Albouy, La création mythologique chez Victor Hugo, p. 170.

(24) V. Hugo, Magnitudo Parvi, vv. 576-577, in La Légende des Siècles, II, p. 242.

(25) P. Albouy, La création mythologique chez Victor Hugo, p. 170.

(26) V. Hugo, Je lisais. Que lisais-je ?, vv. 27-30, in Les Contemplations, p. 173.

(27) P. Albouy, La création mythologique chez Victor Hugo, p. 170.

(28) V. Hugo, Ce que dit la Bouche d'Ombre, v. 613, in Les Contemplations, p. 504. 
paille du cachot! $\gg^{29}$. Con quest'annuncio di redenzione si prepara «la disparition de l'antique anathème» ${ }^{30} \mathrm{e}$ il «retour du banni». ${ }^{31}$ Ritorno e piena consacrazione che avverrà con J'aime l'araignée et j'aime l'ortie (databile attorno alla metà degli anni Cinquanta), dove il ragno diviene protagonista della poesia XXVII del libro III $^{32}$ delle Contemplations ${ }^{33}$. Il testo consacra ufficialmente l'insetto malefico, un tempo semplice apparizione del male, a personaggio centrale della mitologia hugoliana. Il passare degli anni ha indotto inoltre Hugo a operare una fondamentale inversione di giudizio nei riguardi del ragno, "mostro" che non veste più gli esclusivi panni del carnefice e del persecutore ma anche quelli della vittima. Nella poesia è definita per la prima volta nei particolari la fisionomia del ragno, accostato all'ortica, suo corrispettivo vegetale. Esso occupa, assieme alla pianta, il gradino più in basso nella scala degli esseri che popolano il creato. L'aracnide e la pianta sono paria, impuri che portano addosso «l'ombre des abîmes» ${ }^{34}$. L'odio che la comunità universale nutre nei confronti del ragno e dell'ortica è all'origine dell'amore che Hugo prova per le due creature condannate al bando perpetuo, che presentano le tipiche caratteristiche del capro espiatorio, così come appare nelle teorizzazioni del "pensatore della vittima" René Girard. In Des choses cachées depuis la fondation du monde (1978) lo studioso opera una fondamentale distinzione tra l'atteggiamento tenuto dal mito e dal testo biblico riguardo le vittime. Mentre il mito è un resoconto di violenze commesse ai danni di una vittima, "occultate" dalla coscienza mistificatrice dei persecutori, la Bibbia (e in particolar modo il Vangelo) rappresenta il rifiuto dell'ottica persecutoria in favore del punto di vista di colui che subisce la condanna perché considerato "mostro" fisico e / o morale dalla collettività.

Sebbene Hugo non possa proclamare l'assoluta innocenza dei due «misérables», si fa comunque portavoce dei diritti dei maledetti e degli esclusi andando a cercare le ragioni sociali e "naturali" della malvagità degli empi. Ne risulta un'immagine di carnefici al tempo stesso vittime della loro natura inclemente o della società e, in quanto tali, da compiangere. Accanto ad un esasperato manicheismo ${ }^{35}$, è presente paradossalmente in Hugo un rapporto di circolarità tra vittime e carnefici che rende a volte labile il confine tra oppresso ed oppressore: «Le même être est victime et bourreau tour à tour» scrive Hugo nel poema incompiuto Dieu ${ }^{36}$.

Il poeta ama il ragno e l'ortica, neri esseri striscianti, maledetti e disprezzati da tutti perché tristi prigionieri dei loro tranelli, dei «fatals nœuds» ${ }^{37}$ della propria "alienazione". Allo stesso modo in cui i crimini compiuti da Edipo - figura emblematica di «victime émissaire» ${ }^{38}$ presente nella mitologia greca - ricadono sul sovrano ancor prima che sugli abitanti di Tebe, così il male commesso dal ragno e dall'ortica si ritorce anzitutto contro loro stessi. Nella poesia si verifica altresì un parziale rovesciamento della simbologia del ragno impiegata in Notre-Dame de Paris. L'aracnide non è più la personificazione della fatalità ma è anch'esso un essere preso nella tela dell'ananké,

(29) V. Hugo, Ce que dit la Bouche d'Ombre, vv 739, 743-745, in Les Contemplations, p. 508.

(30) Ivi, v. 751, p. 509.

(31) Ivi, v. 753 , p. 509.

(32) Si tratta del libro "Les luttes et les rêves" in cui il poeta denuncia la miseria morale e sociale di cui è testimone (guerra, tirannia, pena di morte etc).

(33) Sulla poetica hugoliana vedi: J. GAUDON, Le Temps de la contemplation, l'oeuvre poétique de Victor Hugo, des Misères au Seuil du gouffre, 1845 1856, Paris, Flammarion, 1969.

(34) V. Hugo, J'aime l'araignée et j'aime l'ortie, v 13, in Les Contemplations, p. 216.
(35) Ovvero «le dualisme des monstres et demidieux» nella cui esasperazione, a detta di Girard, «se situe le génie véritable de Hugo» (R. GIRARD, Monstres et demi-dieux dans l'auvre de Hugo, in «Simposium», XIX, 1, primavera 1965 , p. 57).

(36) V. Hugo, Dieu, in La Légende des Siècles, La Fin de Satan, Dieu, («Bibliothèque de la Pléiade») Paris, Gallimard, 1984, p. 1011.

(37) V. Hugo, J'aime l'araignée et j'aime l'ortie, v 10, in Les Contemplations, p. 216

(38) "CEdipe et la victime émissaire" è il titolo del cap III de La violence et le sacré, (Paris, Grasset, 1972. Nuova edizione: Paris, Hachette, 1998, pp. 105-134) di R. Girard. 
travolto dall'opera malvagia che sta compiendo. Un po' più avanti nel corso di J'aime l'araignée, Hugo designerà esplicitamente col nome di «victimes» le miserabili creature penalizzate da una natura crudele, prigioniere della «sombre nuit $»^{39}$. Di fronte a un tale spettacolo di miseria, il sentimento che domina il poeta non è l'odio bensì la pietà. In modo del tutto analogo alla zingara Esmeralda, "buona samaritana" ${ }^{40}$ mossa a compassione dalle sofferenze di Quasimodo, Hugo implora ai passanti la grazia per la «plante obscure ${ }^{41}$ e per il «pauvre animal» ${ }^{42}$. Nell'esortazione hugoliana a commiserare il brutto e il male è sintetizzato ancora una volta il messaggio di Ce que dit la Bouche d'Ombre: «Oh! qui que vous soyez, qui passez dans ces ombres, Versez votre pitié sur ces douleurs sans fond! ${ }^{43}$.

Una "lezione" evangelica che tornerà nei Misérables, dove Mgr Myriel avrà pietà della «grosse araignée, noire, velue, horrible» ${ }^{44}$ presente nel suo giardino. Il sant'uomo scuserà la crudeltà dell'insetto esclamando: «pauvre bête! ce n'est pas sa faute» ${ }^{45}$. Dietro la descrizione del vescovo non è difficile intravedere la sagoma di Hugo e il suo atteggiamento paternalisticamente benevolo nei riguardi di tutte le creature dell'universo:

Il était indulgent pour la création de Dieu. [...] Les laideurs de l'aspect, les difformités de l'instinct, ne le troublaient pas et ne l'indignaient pas. Il en était ému, presque attendri. Il semblait que, pensif, il en allât chercher, au-delà de la vie apparente, la cause, l'explication ou l'excuse $^{46} \cdot[\ldots]$

Il se penchait sur ce qui gémit et sur ce qui expie. L'univers lui apparaissait comme une immense maladie; il sentait partout de la fièvre, il auscultait partout de la souffrance, et, sans chercher à deviner l'énigme, il tâchait de panser la plaie. Le redoutable spectacle des choses créées développait en lui l'attendrissement; il n'était occupé qu'à trouver pour lui-même et à inspirer aux autres la meilleure manière de plaindre et de soulager; [...].

Il y a des hommes qui travaillent à l'extraction de l'or; lui, il travaillait à l'extraction de la pitié. L'universelle misère était sa mine. La douleur de partout n'était qu'une occasion de bonté toujours ${ }^{47}$.

L'immagine del vescovo Myriel mosso a compassione dal ragno appare così squisitamente hugoliana se si pensa a una ironica definizione che Auguste Vacquerie ${ }^{48}$ dette dello stesso Victor Hugo in Profils et grimaces: «l'ami intime des colimaçons et le galant des araignées ${ }^{49}$. Ed esemplare a questo proposito è il contegno tenuto dal poeta nei confronti del granchio della poesia Je payai le pêcheur qui passa son chemin. Dopo aver acquistato un granchio da un pescatore, la voce che parla in prima

(39) V. Hugo, J'aime l'araignée et j'aime l'ortie, v 16, in Les Contemplations, p. 216.

(40) Il motivo della solidarietà tra emarginati torna con grande frequenza nelle opere di Hugo. $\mathrm{Si}$ pensi alle già citate poesie Le Crapaud (dove l'asino, «élu-maudit» del mondo animale, ha pietà del rospo, altro «damné») Sultan Mourad (dove un sanguinario «maudit» allevia per un istante le sofferenze di un maiale «infecte, immonde», meritandosi per un tale gesto il perdono divino per i suoi crimini) e a una serie di componimenti (come Petit Paul, La confiance du Marquis Fabrice o Souvenir de la nuit du 4) che hanno come protagonisti, ancora una volta, categorie di esclusi quali bambini e vecchi, che si scambiano reciprocamente affetto e sostegno.
(41) V. Hugo, J'aime l'araignée et j'aime l'ortie, v. 17, in Les Contemplations, p. 216.

(42) Ivi, v. 18, p. 216.

(43) V. Hugo, Ce que dit la Bouche d'Ombre, vv. 576-577, in Les Contemplations, p. 503.

(44) V. Hugo, Les Misérables, p. 79.

(45) Ibidem.

(46) Ivi, I, pp. 78-79.

(47) Ivi, I, p. 83.

(48) Auguste Vacquerie (1819-1895) poeta, drammaturgo, giornalista, fratello di Charles (genero di Hugo, tragicamente scomparso insieme alla moglie Léopoldine a Villequier nel 1843) aveva raggiunto il poeta a Jersey nel 1852 .

(49) Cit. in: V. Hugo, Les Contemplations, nota p. 605 . 
persona prende nella sua mano la «bête horrible» ${ }^{50}$, l'《être obscur» ${ }^{51}$ odiato da tutti perché ripugnante e cattivo al pari del ragno e dell'ortica. L'orrendo animale, una volta allontanatosi il pescatore, affonda i denti nella carne del nuovo padrone. La reazione hugoliana di fronte all'offensiva del crostaceo non è una risposta di violenza e di vendetta bensì di compassione e di perdono ${ }^{52}$. Allo stesso modo in cui Esmeralda sulla gogna ha pietà di colui che ha tentato di rapirla e gli offre dell'acqua, così il poeta assolve l'animale che lo ha morso e gli restituisce la libertà: «Vis! et sois béni, pauvre maudit! $\gg^{53}$.

L'atteggiamento pietoso del poeta verso gli esseri malvagi, sofferenti perché vittime di un doloroso destino, si inserisce per Hugo in una prospettiva cosmica di aspirazione all'amore. Ogni creatura vivente anela all'amore e la bruttezza esclude da esso. La cattiveria è il risultato della sofferenza che la disgrazia dell'essere brutto provoca sull'individuo poiché «dans l'absolu, être hideux, c'est haïr» ${ }^{54}$. In realtà «Tout veut un baiser»" ${ }^{55}$, anche la «vilaine bête et la mauvaise herbe / Murmurent: Amour! $\gg^{56}$. Un po' di compassione e di misericordia sono sufficienti per salvare l'anima di qualunque miserabile, dichiara Quasimodo ad Esmeralda ${ }^{57}$. Il cattivo è dunque un debole, un incompreso, un infelice che deve essere perdonato e che attraverso l'amore può riscattarsi.

La completa redenzione del ragno avverrà in Puissance égale bonté (datata 15 novembre 1857). Qui si compie ad opera di Dio la definitiva metamorfosi dell'aracnide in Sole, stella suprema. La poesia della Légende des Siècles ruota attorno alla rivalità che oppone Iblis del Corano, angelo decaduto e genio del male, a Dio. Iblis propone a Dio una gara: il demone prenderà l'opera di Dio e la trasformerà; in seguito l'Onnipotente feconderà l'opera offerta da Iblis. Dio accetta e al termine della competizione trasforma l'insetto in Sole. Si attua così la definitiva "alchimia" del male in bene, dell'ombra in luce ${ }^{58}$, caratteristica della "religione" hugoliana:

Et Dieu prit l'araignée et la mit au milieu

Du gouffre qui n'était pas encore le ciel bleu;

Et l'esprit regarda la bête; sa prunelle,

Formidable, versait la lueur éternelle;

Le monstre, si petit qu'il semblait un point noir,

Grossit alors, et fut soudain énorme à voir;

Et Dieu le regardait de son regard tranquille;

Une aube étrange erra sur cette forme vile;

L'affreux ventre devint un globe lumineux;

Et les pattes, changeant en sphères d'or leurs nœuds,

S'allongèrent dans l'ombre en grands rayons de flamme.

Iblis leva les yeux; et tout à coup l'infâme,

Ébloui, se courba sous l'abîme vermeil;

(50) V. Hugo, Je payai le pêcheur qui passa son chemin, v 2, in Les Contemplations, Paris, Livre de Poche, 1985, p. 354. Il mostruoso granchio comparirà nuovamente nel romanzo Les Travailleurs de la Mer, dove si stablisce, a posteriori, un singolare legame tra il ragno e il crostaceo, il cui inseguimento da parte dell'affamato Gilliatt costituisce la causa immediata della caduta dell'eroe nella trappola della piovra-ragno (V. Hugo, Les Travailleurs de la Mer, pp. 490-493).

(51) V. Hugo, Je payai le pêcheur qui passa son chemin, v. 3, in Les Contemplations, p. 354.
(52) Vedi: V. Hugo, Ce que dit la Bouche d'ombre, v 616: «Pleurez [...] / Sur le crabe hideux» (Les Contemplations, p. 504).

(53) V. Hugo, Je payai le pêcheur qui passa son chemin, v. 14, in Les Contemplations, p. 354.

(54) V. Hugo, Les Travailleurs de la Mer, p. 497.

(55) V. Hugo, J'aime l'araignée et j'aime l'ortie, v. 22, in Les Contemplations, p. 217

(56) Ivi, vv 27-28, p. 217.

(57) V. Hugo, Notre-Dame de Paris, p. 386.

(58) Cfr. P. Lejeune, L'Ombre et la lumière dans "Les Contemplations" de Hugo, Paris, Minard, 1969. 
Car Dieu, de l'araignée, avait fait le soleil ${ }^{59}$.

Alla fine del poema, che oppone l'azione provvidenziale all'azione satanica, l'odio all'amore (confermando il ruolo essenziale giocato dal male nell'opera divina) Dio ha benedetto il maledetto trasformandolo in Sole. «Dans ce poème - afferma Albouy - l'ananké a trouvé sa fin dans la rédemption» ${ }^{60}$. Con questo mito Hugo ha inoltre illustrato i dogmi essenziali del suo "credo": «la transfiguration du mal et de l'illumination de l'ombre» ${ }^{61}$ (già presenti in Ce que dit la Bouche d'Ombre) e la reintegrazione del male in Dio (pensiero guida de La Fin de Satan).

Il Sole è dunque per Hugo un'immagine divina. Sotto un profilo strettamente antropologico la metamorfosi del ragno in Sole ricorda molto il processo di sacralizzazione al quale vengono sottoposte, dopo l'immolazione, le vittime presenti nelle mitologie di tutti i popoli della Terra. Il mutamento dell'aracnice in astro pare riprodurre, all'interno della mitologia hugoliana ${ }^{62}$, il meccanismo vittimario di condanna e riabilitazione di un capro espiatorio, spia significativa dei sentimenti ambivalenti del poeta. Il duplice "movimento" di identificazione con il carnefice (o con la comunità dei persecutori) e, successivamente, con la vittima, trova una articolata e illuminante disamina nel saggio Monstres et demi-dieux dans l'œuvre de Hugo ${ }^{63}$ (1965), analisi dedicata da Girard al tema della mostruosità nella creazione hugoliana. Secondo l'interpretazione fornita dal critico, il pullulare di immagini mostruose nell'opera di Hugo sarebbe la conseguenza della sconfitta del desiderio "metafisico" del grande Olympio nel suo tentativo di volersi elevare a unica divinità dell'Olimpo letterario europeo. Fin dalla giovinezza Hugo «crée des monstres ${ }^{64}$ e continuerà per tutta la vita a riempire di creature difformi le pagine delle sue opere in prosa e in versi. Quasimodo, Gwynplaine, il rospo, il ragno sono mostri. Ma sono anche, in ultima analisi, vittime: della natura e / o della società. Se i mostri degli anni Venti e Trenta (Habibrah, Triboulet, Quasimodo) rispettano ancora, sebbene in parte, i caratteri del mostro tradizionale (ossia il topos della malvagità associata alla deformità) i mostri degli anni Cinquanta e Sessanta (Gwynplaine, il rospo) rovesciano l'immagine consueta della bontà legata alla bellezza perché appaiono, paradossalmente, vittime dei sedicenti "normali", degli umani "demi-dieux". Girard vede nel processo di riabilitazione del mostro un «processus d'identification» ${ }^{65}$. Hugo si identificherebbe con i mostri per lamentarsi dei suoi simili che lo perseguitano ingiustamente (lo scrittore è, in quegli anni, esule a causa di Napoléon III), per proclamare la propria innocenza e non rinunciare al desiderio luciferino di essere il "Re Sole" della letteratura. È noto che Hugo nutrì sempre sconfinate ambizioni letterarie: «Je veux être Chateaubriand ou rien» scrive il giovane Victor il 10 luglio $1816^{66}$. Egli scopre tuttavia di non essere l'unico astro del

(59) V. Hugo, Puissance égale Bonté, vv 76-92, in La Légende des Siècles, I, pp. 90-91. L'immagine del ragno illuminato, sebbene non ancora trasfigurato dalla luce del sole, era apparsa nella poesia Dieu est toujours là (datata 1837 e inclusa nella raccolta Les voix intérieures) dedicata alla Provvidenza divina che veglia sul povero: «Quand l'été vient, le pauvre adore! / L'été, c'est la saison de feu, / C'est l'air tiède et la fraîche aurore; / L'été c'est le regard de Dieu. [...] L'aube alors de clartés baignée, / Entrant dans le réduit profond, / Dore la toile d'araignée / Entre les poutres du plafond.» (Dieu est toujours là, vv. 1-4, 41-44, in Les voix intérieures, in V. Hugo, Euvres complètes, Poésie I, Paris, Robert Laffont, pp. 832-833). Cfr: S. Ballestra-Puech, «Car Dieu, de l'Araignée, avait fait le soleil: Métamorphose d'Arachné chez Victor Hugo», in Victor Hugo ou les frontières effacées, textes réunis par Dominique Peyrache-Leborgne et Yann Jumelais, Nantes, Editions Pleins Feux («Horizons Comparatistes. Université de Nantes») 2002, pp. 107-122.

(60) P. Albouy, La création mythologique chez Victor Hugo p. 171.

(61) Ibidem.

(62) Si veda in merito: P. Albouy, Mythographies, Paris, José Corti, 1976.

(63) Pubblicato in: «Simposium», XIX, 1, primavera 1965, pp. 50-57; ripreso in: R. GIRARD, Critique dans un souterrain, Lausanne, l'Age d'Homme, 1976.

(64) R. GIRARD, Monstres et demi-dieux dans l'œuvre de Hugo, p. 50.

(65) Ivi, p. 52.

(66) A. Maurois, Olympio ou la vie de Victor 
firmamento artistico nazionale ed internazionale. «Son orgueil - scrive Girard - n'admet pas le partage et il abandonne à autrui ce royaume de la forme, de la lumière et de l'harmonie où il découvre qu'il n'est pas seul à régner, il se précipite donc dans le domaine de l'informe, de l'obscurité et du désordre; il se réfugie parmi les monstres et il se confond avec eux» ${ }^{67}$.

Hugo si fa mostro perché in precedenza si era identificato con Dio. Nel momento in cui il poeta scopre di non essere l'unica divinità dell'universo letterario ${ }^{68}$, regno di luce e d'armonia, rifiuta i limiti imposti alla sua potenza e si confonde con i mostri che abitano i regni infernali del "sottosuolo". Ora, Hugo è profondamente legato alle immagini classiche e tutto ciò che non è armonia appare ai suoi occhi difforme. L'orgoglio non vuole abdicare e la sconfitta del desiderio prende l'aspetto della mutilazione, della mostruosità, dell'animalità. Hugo «se découvre monstre mais il cherche alors à diviniser le monstre car il ne cesse pas, monstre, de se vouloir dieu» ${ }^{69}$. Si spiega così, secondo Girard, l'«epifania mimetica $>^{70}$ di mostri e di animali nell'opera dello scrittore, espressione estetica che rivela il fondamento esistenziale del satanismo di $\mathrm{Hugo}^{71}$ :

Dans tout rapport intensément mimétique, le sujet s'efforce de combattre le mépris de soi qui accompagne nécessairement la surestimation du médiateur. [...] Les images de bestialité sont le véhicule de l'abaissement de soi qu'engendre le désir. Au lieu de se hisser à l'étage quasi divin où ils situent leurs modèles, les sujets désirants glissent vers l'animalité ${ }^{72}$.

Girard definisce il momento dell'identificazione con i mostri «le moment "masochiste" de la conscience de Hugo» ${ }^{73}$. Tale momento, però, «est précédé d'un moment "sadique" " $^{74}$ in cui l'autore si era identificato con i "demi-dieux", con i Lords che deridono Gwynplaine, con i monelli che torturano il rospo, con il genere umano che aborrisce il ragno.

Esiste, secondo Girard, un preciso "percorso" artistico-esistenziale ${ }^{75}$, individuabile in molte opere hugoliane e applicabile perfettamente al motivo del ragno. L'esame dei testi pare confermare questo processo. Hugo, all'inizio della sua opera, "immola" il ragno vedendo in esso un essere orrido e malvagio, dopodiché, col passare degli anni, «s'identifie à sa victime et la réhabilite sans mesure» ${ }^{76}$, finendo per "beatificare" l'insetto che aveva in precedenza "giustiziato", trasfigurandolo nell'immagine suprema della bellezza e dell'amore di Dio: il Sole.

FEDERICA CASINI

Hugo, Paris, Hachette, 1954, p. 60. Cfr: R. JeAN, "Chateaubriand ou rien", in "Cahiers du Sud», n. 50, 1960 , pp. $207-212$.

(67) R. GIRARD, Monstres et demi-dieux dans l'cuvre de Hugo, p. 53.

(68) Si può affermare ragionevolmente che Goethe sia uno degli "dei" / dell'Olimpo letterario europeo contro cui si rivolge la reazione di Hugo. Si ricordi a questo proposito il duro giudizio che lo scrittore tedesco espresse a proposito di Notre-Dame de Paris in una conversazione con Eckermann, giudizio applicabile all'intera produzione hugoliana. Proiettato, col suo Secondo Faust, verso gli ideali di bellezza classica Goethe non può più sopportare in Hugo «la fâcheuse direction romantique ... qui le pousse à représenter ... les choses ... les plus laides» (V. Hugo, Notre-Dame de Paris, Les Travailleurs de la mer, Textes établis, présentés et annotés par J. Seebacher et Y. Gohin, Paris, Gallimard, 1975, note p. 1069).

(69) R. GIRARD, Monstres et demi-dieux dans l'auvre de Hugo, p. 54.

(70) Cfr: A.-M. Scaiola, Epifanie mostruose. Una produzione del «grottesco» in Hugo, in Metamorfosi, mostri, labirinti (Atti del seminario di Cagliari, 22-
24 genn. 1990) Roma, Bulzoni, 1991, pp. 83-96; il numero monografico della rivista «Le français dans tous ses états», $\mathrm{n}^{\circ} 49,2002$, dal titolo Le monstre dans l'oeuvre de Victor Hugo.

(71) R. GIRARD, Monstres et demi-dieux dans l'œuvre de Hugo, p. 55. Vedi anche: P. ZumTHOR, Victor Hugo, poète de Satan, Paris, Robert Laffont, 1946 (ristampa: Genève, Slatkine, 1973).

(72) R. GIRARD, Shakespeare. Les feux de l'envie, Paris, Livre de Poche, 1990, p. 88.

(73) R. GIRARD, Monstres et demi-dieux dans l'œuvre de Hugo, p. 54.

(74) Ibidem. Cfr. C. НАвIB, Le sadisme et la pitié, in «Revue des lettres modernes», 1024-1032, 1991, pp. 111-123.

(75) Percorso sicuramente interessante che non esaurisce, tuttavia, il problema dell' "autenticità" dell'ispirazione hugoliana nei confronti del tema della vittima, che non può essere ridotta a puro tentativo autogiustificatorio ma deve essere considerata una delle espressioni più mature del suo complesso ed eterodosso cristianesimo.

(76) R. GIRARD, Monstres et demi-dieux dans l'œuvre de Hugo, p. 54. 